\title{
Study on the multi-objective optimization of reliability and operating cost for natural gas pipeline network
}

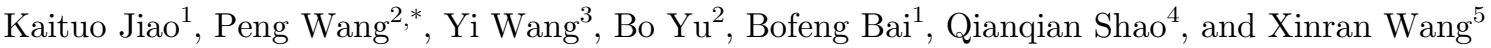 \\ ${ }^{1}$ State Key Laboratory of Multiphase Flow in Power Engineering, Xi'an Jiaotong University, Xi'an 710049, PR China \\ ${ }^{2}$ School of Mechanical Engineering, Beijing Key Laboratory of Pipeline Critical Technology and Equipment for Deepwater Oil \& Gas \\ Development, Beijing Institute of Petrochemical Technology, Beijing 102617, PR China \\ ${ }^{3}$ National Engineering Laboratory for Pipeline Safety/MOE Key Laboratory of Petroleum Engineering/Beijing Key Laboratory \\ of Urban Oil and Gas Distribution Technology, China University of Petroleum, Beijing 102249, PR China \\ ${ }^{4}$ School of Petroleum Engineering, Guangdong University of Petrochemical Technology, Maoming 525000, PR China \\ ${ }^{5}$ National Oil and Gas Exploration and Development Company Ltd., Beijing 100034, PR China
}

Received: 19 November 2020 / Accepted: 9 April 2021

\begin{abstract}
The development of natural gas pipeline network towards larger scale and throughput has urged better reliability of the pipeline network to satisfy transportation requirement. Previously, studies of optimizing natural gas pipeline network have been mainly focused on reducing operating cost, with little concern on the reliability of pipeline network. For a natural gas pipeline network with a variety of components and complicated topology, a multi-objective optimization model of both reliability and operating cost is proposed in this study. Failure of each component and the state of pipeline network under failure conditions are taken into account, and minimum cut set method is employed to calculate the reliability of the pipeline network. The variables to be determined for the optimization objectives are the rotating speed of compressors and the opening of valves. Then the solving procedure of the proposed model is presented based on Decoupled Implicit Method for Efficient Network Simulation (DIMENS) method and NS-saDE algorithm. The validity of the optimization model is ascertained by its application on a complicated pipeline network. The results illustrate that the optimization model can depict the relative relationship between reliability and operating cost for different throughput, by which the operation scheme with both satisfying reliability and operating cost can be obtained. In addition, the customer reliability and the impact of the failure of each pipeline on the whole network can be evaluated quantitatively to identify the consumers and pipelines of maintenance priority. The pipeline network reliability can be improved through proper monitoring and maintenance of these consumers and pipelines.
\end{abstract}

\section{Nomenclature}

$c_{\mathrm{p}} \quad$ Specific heat capacity at constant pressure, $\mathrm{J} /(\mathrm{kg} \mathrm{K})$

$c_{\mathrm{v}}$

$\mathrm{CR}$

$d$

$D$

$D_{\mathrm{i}}$

F

FR

$g$

G
$H$

HA

$K$

$K_{\mathrm{e}}$

$L$

$m$

$\mathrm{MC}$

$n$

$N$

$\mathrm{Nc}$

$\mathrm{NP}$

$p$

$P$

$q$

\footnotetext{
* Corresponding author: wangp@bipt.edu.cn
}

Pressure head provided by a compressor, $\mathrm{m}$ Hydraulic Availability Total heat transfer coefficient, $\mathrm{W} /\left(\mathrm{m}^{2} \mathrm{~K}\right)$

Pipe absolute roughness, $\mathrm{mm}$

Pipeline length, $\mathrm{m}$

Mass flow rate, $\mathrm{kg} / \mathrm{s}$

The minimum cut set

Rotational speed of a compressor, $\mathrm{r} / \mathrm{min}$

Number of components

Number of consumers

Population size

Polytropic index

Pressure, $\mathrm{Pa}$

The individual which represents an operation scheme 


$\begin{array}{ll}Q & \text { Volume flow rate }, \mathrm{m}^{3} / \mathrm{s} \\ R a & \text { Specific gas constant of air, } \mathrm{J} /(\mathrm{kg} \mathrm{K}) \\ R j & \text { Pipeline network reliability of the system } \\ \mathrm{Re} & \text { Reynolds number } \\ t & \text { Period considered, } \mathrm{h} \\ \mathrm{Sc} & \text { Number of compressors } \\ T & \text { Temperature, } \mathrm{K} \\ T_{\mathrm{a}} & \text { The ambient temperature, } \mathrm{K} \\ V & \text { Specific volume, } \mathrm{m}^{3} / \mathrm{kg} \\ v_{q}, G & \text { Mutation vector } \\ x_{q}, G & \text { Decision vector } \\ \mathrm{z}_{q}, G & \text { Trial vector } \\ Z & \text { Compressibility factor } \\ Z F & \text { Amplification factor of difference vector }\end{array}$

\section{Greek symbols}

$\begin{array}{ll}\beta & \text { Transmission coefficient } \\ \delta_{\mathrm{p}} & \text { Failure probability of a pipeline, }(\mathrm{km} \mathrm{h})^{-1} \\ \varepsilon & \text { Compression ratio } \\ \phi_{\mathrm{k}} & \text { State probability of component } k \\ \phi(\mathrm{MC}) & \text { Occurrence probability of MC } \\ \eta & \text { Efficiency of a compressor } \\ \lambda & \text { Friction factor } \\ v & \text { Kinematic viscosity, } \mathrm{m}^{2} / \mathrm{s} \\ \rho & \text { Density, } \mathrm{kg} / \mathrm{m}^{3} \\ \zeta & \text { Number of the minimum cut sets } \\ \Delta & \text { Specific density of natural gas relative to air } \\ \theta & \text { Heat transfer coefficient }\end{array}$

\section{Superscripts}

$\begin{array}{ll}\text { req } & \text { Required } \\ \min & \text { Minimum } \\ \max & \text { Maximum } \\ \text { given } & \text { Known value }\end{array}$

\section{Subscripts}

$\begin{array}{ll}* & \text { Standard state } \\ j & \text { Index of consumer } \\ k & \text { Index of component } \\ \text { in } & \text { Inlet } \\ \text { out } & \text { Outlet }\end{array}$

\section{Introduction}

The worldwide concern about fuel pollution and the urgent need to upgrade energy structure mean that natural gas as a clean fuel plays a more important role in energy consumption. In the year 2018, global consumption of natural gas gained the fastest growth since 1984 by an amount of 195 billion cubic meters with a growth rate of $5.3 \%$ [1]. It is predicted that global consumption of natural gas will keep at an annual growth rate of $2.9 \% \sim 3.2 \%$ until the year 2030 [2]. There is no doubt that natural gas industry will continue to develop rapidly in the future [3]. Generally, natural gas sources are far from the consumption sites, and the transportation is realized by natural gas pipeline network which extends over larger and larger areas along with the increase of natural gas consumption. As a result, safe and efficient operation of the pipeline network become more important than at any time in the past. Besides, characterized with fluctuating gas consumption and limited allowable pressure, natural gas pipeline network may easily operate deviating from the optimal condition. Therefore, conducting research to determine the optimal operation scheme is extremely essential for the proper and smooth running of natural gas pipeline network.

Based on the number of optimization objectives, the natural gas pipeline network optimization can be classified into single-objective optimization and multi-objective optimization. The operation of natural gas pipeline network consumes huge amount of energy, with compressors accounting for $25 \% \sim 50 \%$ of the pipeline company's total operating budget [4] and $10 \%$ of the industrial electricity cost [5]. As a result, previous researches have focused on single-objective optimization to reduce the operating cost [6-8]. However, aspects such as safety, environmental protection and stability of natural gas pipeline network are all important factors besides operating cost. In recent years, multi-objective optimization has obtained more and more applications in oil and gas industry due to its comprehensive consideration of multiple motivations. For complex pipeline network structures, Botros et al. [9] adopted a multiobjective optimization model to study simultaneous optimization of operating cost and line-pack of natural gas pipeline network. Kashani and Molaei [10] proposed a three-objective optimization model to optimize the operation parameters for low throughput, reduced operating costs and $\mathrm{CO}_{2}$ emissions. Panda and Ramteke [11] performed multi-objective optimization of oil transmission network scheduling to account for the transportation profit and flow fluctuation in an oil distillation unit. Many researchers have devoted efforts to multi-objective optimization of compressor and pump station $[4,12,13]$ in terms of the power or fuel consumption of the stations, the number of stations and the outlet pressure of stations. In addition, considering the gas consumption for electricity generation, there has been combined multi-objective optimization of the gas network and the electricity network [14-16]. Multi-objective optimization has also been applied to pipeline-related equipment, such as sensor placement in the network [17], mini-Liquefied Natural Gas (LNG) plant [18] and gas drainage system [19]. Based on the references mentioned above, the optimized effect is greatly influenced by the optimization algorithm. The evolutionary algorithms are preferred due to their excellent capability of global search. Among the kinds of evolutionary algorithms, the Differential Evolution (DE) algorithm is proved to perform best [20, 21]. It can 
overcome the difficulty in determining the value of algorithm control parameters using the Self-adapting approach [22]. To realize the multi-objective strategy, the DE algorithm can also combine with the Elitist Non-dominated Sorting method, named NS-DE algorithm, which has excellent convergence performance $[23,24]$.

During the operation of natural gas pipeline network, some unpredictable reasons, such as erosion, misguided use and quality defects, may threaten normal gas supply. The ability of the pipeline network to safely and stably complete the transportation task is referred to as the pipeline network reliability, which is tightly related to the topology, equipment status and transportation load of the pipeline network [25]. Study on the reliability of pipeline network is necessary to ensure safe and stable transportation and minimize the loss caused by component failure $[26,27]$. Generally speaking, the pipeline network reliability declines as the power consumption of the compressor is reduced through traditional economic optimization. This is because a pipeline optimization scheme with low operating cost usually has relatively low internal pressure, which cannot provide sufficient energy to ensure the gas flow in the pipeline in failure situations of the pipeline network. Shinstine et al. [28] performed analysis of water supply network reliability, and it was found that reliability has a negative relationship with both the throughput and the pressure demand of consumers. Yu et al. [29] proposed that pipeline network reliability would decrease with increasing probability of the flow rate reaching extreme values. Therefore, operation scheme with best pipeline network reliability and economy is impossible, but instead balancing between reliability and economy is necessary.

There have been a limited number of researches considering reliability in the optimization of natural gas pipelines. The fundamental reason is that the traditional method to calculate the reliability of gas pipeline network, namely the Monte Carlo method [25, 27], is not suitable for the optimization due to its massive calculation. Therefore, the analytical methods are used to represent some characteristics of reliability in the following literature. To trade off the reliability and power demand, Su et al. [26] developed a multi-objective optimization method of minimizing power demand and the risk of gas supply shortage. The reliability is calculated by limit function, and sensitivity analysis is performed to demonstrate the influence of demand uncertainties on the optimization results. Li and Liu [30] used the recursive decomposition method to analyze the seismic capability reliability and seismic connectivity reliability for the water distribution system and gas network. Using the scenario reduction algorithm, Shabazbegian et al. [31] took into account the contingency analysis and role of flexibility options in the optimization process to increase gas supply stability. Moreover, the minimum cut-set method, which has been successfully applied to water supply network [32] but not found in the optimization of gas pipeline, is also an analytical method to evaluate the reliability of pressure supply. However, we found the following limitations from the literature review: (1) The failure possibility of multi-components was neglected in previous reliability optimization. (2) Reliability calculation has been restricted to the assumption of simple network topology. (3) The reliability was considered to have little relationship with the hydraulic-thermal state of fault pipeline network containing failed components.

To address the above issues, this paper proposes a multi-objective optimization model considering both reliability and operating cost, applicable to natural gas network with multi-source, multi-consumer, multiple components and complicated topology. In the optimization process, the fault pipeline network involving failed components is considered, and the hydraulic-thermal state of the fault pipeline network is included into the reliability evaluation by adopting the minimum cut-set method. By using the presented model, the relationship balancing between pipeline network reliability and economical consideration is investigated in detail for the nature gas pipeline network under different throughputs.

The remaining of the paper is organized as follows: Section 2 presents the introduction of reliability and governing equations of the pipeline network. Then, the multiobjective optimization model of reliability and operating cost for natural gas pipeline network operation is proposed in Section 3. The solving method of the model and the overall calculation procedure are illustrated in Section 4. The cases, optimal results and discussion are given in Section 5 . The final conclusion is drawn in Section 6 .

\section{Reliability and governing equations}

\subsection{Reliability of the pipeline network}

The optimization of complex gas pipeline network involves evaluation of a large number of operation schemes, meaning that effective and quick evaluation of each scheme is necessary. The minimum cut-set method is a favorable choice for pipeline network optimization due to its ability of comprehensively assessing the pipeline network with a small amount of calculation. Also the minimum cut-set method is applicable to the reliability evaluation of natural gas pipeline network with multi-components and complicated topology.

In this subsection, the minimum cut-set method is introduced [33]. Provided that there is a set of components in a network system, which when failed causes the failure of this system. While, when any component of the set doesn't fail, the failure of the network system does not occur. Then, this set of components is the minimum cut set of the network system. The minimum cut set of the pipeline network is the combination of different number of pipelines, valves and compressors. The minimum cut set is determined by whether the demand of consumers in the system is satisfied, that is whether the pressure or gas flow rate reaches the required level. Thus, the evaluation of consumer reliability is first performed through its corresponding minimum cut sets, and then the reliability of the pipeline network can be evaluated.

Here we assume consumer $j$ has $\zeta_{j}$ minimum cut sets, and the ith minimum cut set is $\mathrm{MC}_{i}$ which has $N$ components. When all the components are in failure status, 
$\mathrm{MC}_{i}$ occurs. Then the occurrence probability of this minimum cut set can be written as:

$$
\phi_{j}\left(\mathrm{MC}_{i}\right)=\prod_{k=1}^{N} \phi_{k} .
$$

For consumer $j$, the failure probability and the consumer reliability are respectively described as:

$$
\begin{gathered}
F_{j}=\sum_{i=1}^{\zeta_{j}} \phi_{j}\left(\mathrm{MC}_{i}\right), \\
R_{j}=1-F_{j}=1-\sum_{i=1}^{\zeta_{j}} \phi_{j}\left(\mathrm{MC}_{i}\right) .
\end{gathered}
$$

In a gas transmission project, the gas volume supplied to one consumer is related to the pressure at this consumer, and thus we consider the influence of the pressure at consumers on the pipeline network reliability. Therefore, hydraulic availability is introduced [34]:

$$
\mathrm{HA}_{j}\left\{\begin{array}{cc}
0 & P_{j}<P_{j}^{\min } \\
\sqrt{\frac{P_{j}-P_{j}^{\min }}{P_{j}^{\text {req }}-P_{j}^{\min }}} & P_{j}^{\min }<P_{j}<P_{j}^{\mathrm{req}} . \\
1 & P_{j}>P_{j}^{\mathrm{req}}
\end{array}\right.
$$

It should be noted that, the pressure is closely related with the hydraulic-thermal state of the fault pipeline network, obtained by solving the hydraulic equations and thermodynamic equations, which is introduced in the next section.

The hydraulic availability can better describe the gas supply ability of the pipe network under abnormal conditions, by introducing which, the reliability at consumer node $j$ is redefined as:

$$
R_{j}=1-\sum_{i=1}^{\zeta_{j}}\left(1-\mathrm{HA}_{j}^{i}\right) \phi_{j}\left(\mathrm{MC}_{i}\right)
$$

In equation (5), the $(1-\mathrm{HA})$ can be regarded as the hydraulic unavailability of a consumer node. Thus, the occurrence probability of $\mathrm{MC}_{i}$ is multiplied by $\left(1-\mathrm{HA}_{j}^{i}\right)$ to formulate the reduction degree of the gas supply ability to consumer $j$. Therefore, by equation (5), a continuous hydraulic availability concept is used.

After calculating the hydraulic reliability of each consumer node, the gas supply reliability of the pipeline network can be calculated by weight factor method as the weighted average of all the consumer nodes. And the consumption amount of each consumer node acts as the weighted value. The pipeline network reliability can be calculated by:

$$
R_{s}=\frac{\sum_{j=1}^{N_{C}} m_{j}^{\mathrm{req}} R_{j}}{\sum_{j=1}^{N_{C}} m_{j}^{\mathrm{req}}} .
$$

\subsection{Governing equations}

The pipeline network is a complex system, the pressure, flow rate, and temperature of which satisfy certain governing equations including hydraulic equation, thermodynamic equation, node equation and gas state equation. For convenience, we involve two assumptions: (1) The pipeline network is simplified as a structure composed of nodes and components. Pipelines, valves and compressors are seen as components, while sources, consumers and componentto-component connections are nodes. (2) The pipelines are horizontal and the flow in the pipe network is steady.

\subsubsection{Hydraulic equations}

The hydraulic equations describe the relationship between flow rate and pressure drop of the components. For the pipelines, valves and compressors, the hydraulic equations are presented as follows.

Hydraulic equation of a pipeline with gas flow [35, 36]:

$$
P_{\text {in }}^{2}-P_{\text {out }}^{2}=\frac{\lambda Z \Delta T L}{C_{*}^{2} d^{5}} Q_{*}^{2},
$$

where $C_{*}=\frac{\pi}{4} \frac{\sqrt{R_{a}} T_{*}}{P_{*}}$.

Hydraulic equation of a compressor [35]:

$$
P_{\text {out }}-\varepsilon P_{\text {in }}=0 \text {. }
$$

Hydraulic equation of a valve [37]:

$$
Q_{\text {in }}-C v \sqrt{\frac{\left(P_{\text {in }}^{2}-P_{\text {out }}^{2}\right)}{Z \Delta T_{\text {in }}}}=0,
$$

where $C v=f(F R)$.

\subsubsection{Thermodynamic equations}

The thermodynamic equations mainly describe the temperature variations of components, which are provided below for the pipelines, valves, and compressors respectively.

Thermodynamic equation of a pipeline [35]:

$$
\begin{aligned}
T_{\text {out }}= & T_{a}+\left(T_{\text {in }}-T_{a}\right) \mathrm{e}^{-\theta L}-D_{i} \\
& \times \frac{P_{\text {in }}-P_{\text {out }}}{\theta L}\left(1-\mathrm{e}^{-\theta L}\right),
\end{aligned}
$$

where $\theta=\frac{K \pi D}{m c_{p}}$.

Thermodynamic equation of a compressor [37]:

$$
T_{\text {out }}-T_{\text {in }} \varepsilon^{\frac{p-1}{p}}=0 .
$$

Thermodynamic equation of a valve [37]:

$T_{\text {out }}-T_{\text {in }}+\left(P_{\text {out }}-P_{\text {in }}\right)\left\{\frac{1}{c_{p}}\left[\frac{T}{\rho^{2}} \frac{(\partial P / \partial T)_{\rho}}{(\partial P / \partial \rho)_{T}}-\frac{1}{\rho}\right]\right\}_{\text {out }}=0$. 


\subsubsection{Node equations}

According to mass conservation, the mass entering a node is equivalent to the mass flowing out of that node, expressed as:

$$
\sum m_{\text {in }}=\sum m_{\text {out }}
$$

The temperature of natural gas flowing out of a node is calculated by [37]:

$$
T_{\text {out }, 1}=T_{\text {out }, 2}=\ldots=\frac{\sum\left|c_{p} m T\right|_{\text {in }}}{\sum\left|c_{p} m\right|_{\text {out }}} .
$$

Gas is pushed into the network through the source node, while released from the network at the consumer node. External boundary conditions associated with pressure, flow rate and temperature are applied to the source node and the consumer node as follows [37, 38]:

$$
\begin{aligned}
& P=P^{\text {given }}, \\
& m=m^{\text {given }}, \\
& T=T^{\text {given }} .
\end{aligned}
$$

\subsubsection{Gas state equations}

Natural gas is a mixture of many components. The relationships (including partial differential relationships) among $P$, $T$ and $\rho$ of the components are obtained by the BenedictWebb-Rubin-Starling (BWRS) equation [39]:

$$
\begin{aligned}
P= & \rho R T+\left(B_{0} R T-A_{0}-\frac{C_{0}}{T^{2}}+\frac{D_{0}}{T^{3}}-\frac{E_{0}}{T^{4}}\right) \rho^{2} \\
& +\left(b R T-a-\frac{d}{T}\right) \rho^{3}+\alpha\left(a+\frac{d}{T}\right) \rho^{6} \\
& +\frac{c \rho^{3}}{T^{2}}\left(1+\gamma \rho^{2}\right) \exp \left(-\gamma \rho^{2}\right),
\end{aligned}
$$

where $A_{0}, B_{0}, C_{0}, D_{0}, E_{0}, a, b, c, d, \alpha$, and $\gamma$ are the coefficients, and for their calculation refer to Ref. [39].

Generally, the gas flow in a pipeline is turbulent, and the F. Colebrook formula is used to calculate the friction factor:

$$
\frac{1}{\sqrt{\lambda}}=-2 \lg \left(\frac{K_{\mathrm{e}}}{3.7 d}+\frac{2.51}{\operatorname{Re} \sqrt{\lambda}}\right)
$$

where $\operatorname{Re}=\frac{4 \mathrm{Q}}{\pi \mathrm{d} v}$.

The following formula is used to calculate the JouleThomson coefficient [36]:

$$
D_{\mathrm{i}}=\frac{1}{c_{\mathrm{p}}}\left(T\left(\frac{\partial V}{\partial T}\right)_{P}-V\right) .
$$

\section{The multi-objective optimization model}

\subsection{Objective functions}

1. Pipeline network reliability

In the process of optimization, better reliability of the gas supply system is preferred, and thus in terms of network reliability, the optimization objective is:

$$
\max \left\{R_{S}\right\} .
$$

\section{Total power of compressors}

For a natural gas pipeline network, most of the operating cost comes from the compressors, and reducing the total power of the compressors can effectively decrease the operating cost. In this study, the compressor power is used to represent the operating cost of the pipeline system. From the aspect of operating cost, the optimization objective is expressed as:

$$
\min \left\{\sum_{k=1}^{S_{C}} m_{k} \frac{\left(P_{\text {out }}-P_{\text {in }}\right)_{k}}{\eta_{k}}\right\} .
$$

\subsection{Constraints}

\section{Restriction of decision variables}

This paper focuses on the optimization of natural gas pipeline network from the operation perspective. Therefore, the decision variables to be determined for optimization include the compressor rotational speed $n$ and the valve opening FR. Considering practical engineering, the two parameters are bounded as follows:

$$
\begin{gathered}
n^{\text {min }} \leq n \leq n^{\text {max }}, \\
\mathrm{FR}^{\text {min }} \leq \mathrm{FR} \leq \mathrm{FR}^{\text {max }} .
\end{gathered}
$$

\section{Volume flow rate of compressors}

The flow in the compressor is restricted between the surge flow (minimum flow) and the block flow (maximum flow), and thus the flow rate of each compressor has the following constraint:

$$
Q^{\min } \leq Q_{\text {in }} \leq Q^{\max } .
$$

3. Outlet temperature of compressors

The outlet temperature of compressors should not exceed the limit value to protect the insulating layer [36]:

$$
T_{\text {out }}^{\text {compressor }} \leq T^{\max } .
$$

4. Node pressure of the pipeline network

The node pressure in the pipeline network is restricted as:

$$
P^{\min } \leq P \leq P^{\max } .
$$




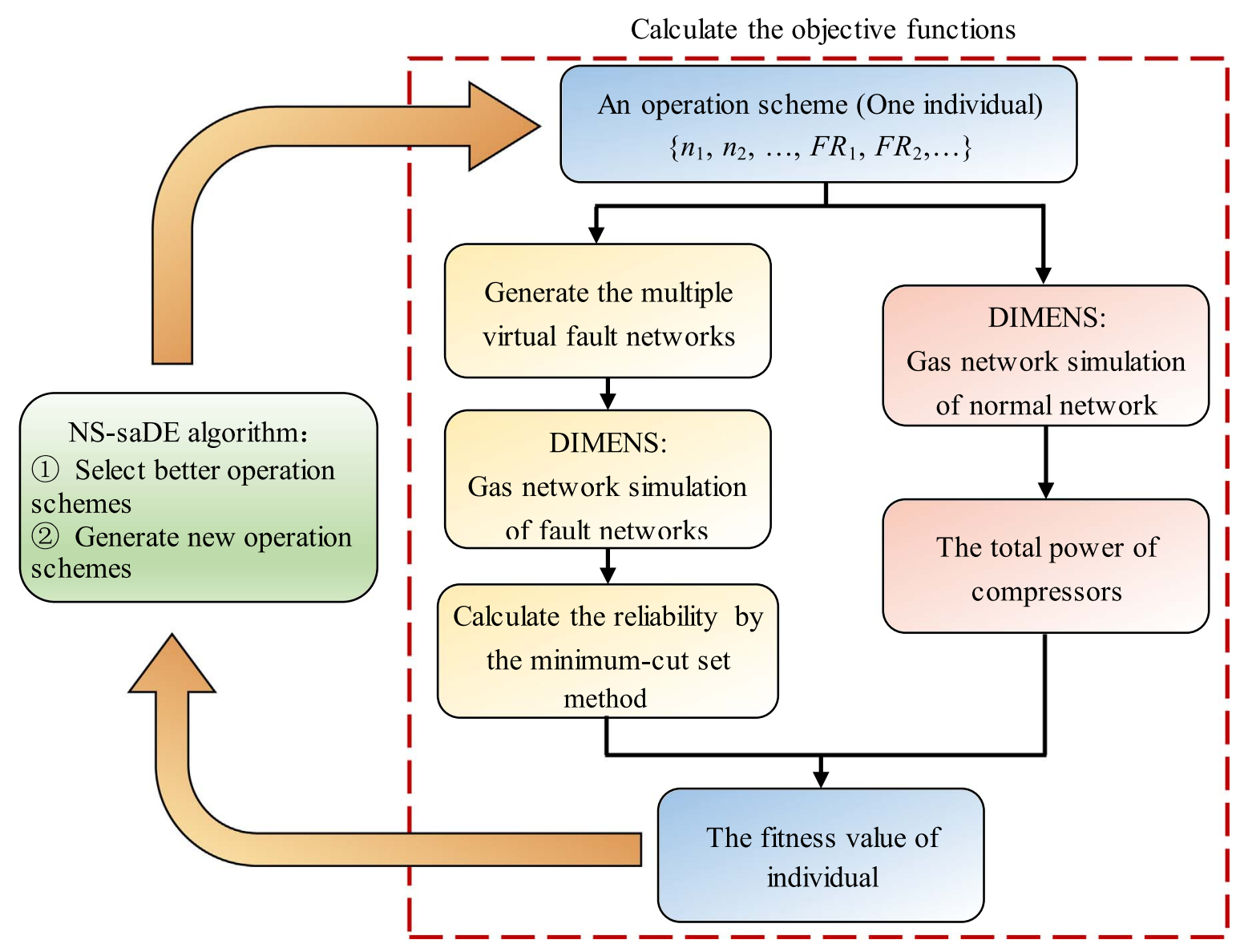

Fig. 1. The overall calculation process.

Equation (27) is applicable to the inlet and outlet pressure of all components, including pipeline, valve and compressor.

\section{Transmission coefficient}

Transmission coefficient $\beta$ is defined for each consumer node to characterize the throughput of the pipeline network:

$$
\beta=\frac{m_{1}^{\mathrm{req}}}{m_{1}^{0}}=\ldots \frac{m_{j}^{\mathrm{req}}}{m_{j-1}^{0}}=\ldots \frac{m_{N_{C}}^{\mathrm{req}}}{m_{N_{C}}^{0}} .
$$

\section{Solution methods}

The overall calculation process is presented in Figure 1. Aimed at optimizing the operation scheme of natural gas pipeline network, operation parameters are the variables to be determined, including the rotation speed of each compressor and the valve opening of each valve. The optimal operation scheme is determined by the combination of compressor speed and valve opening. The optimization has two objectives, one is good pipeline network reliability, and the other is relatively low total power of the compressors. The optimization process consists of two modules, one module to make evaluation of an operation scheme, that is, to calculate the optimization objectives of this operation scheme and get the fitness value of individuals, the other to generate new operation schemes and select better operation schemes. The two modules are nested with each other to form an iteration of calculation. The optimization process proceeds until a planned number of iterations.

When calculating the pipeline network reliability, the components in the pipeline network are assumed to fail one by one, in which a multiple of virtual fault networks is firstly generated. Then, the hydraulic-thermal state of the various fault networks is obtained by the gas network simulation. Thus, the pipeline network reliability can be quantified by the minimum cut-set method. When calculating the total power of compressors, the hydraulic-thermal state of the normal network is also determined by gas network simulation.

To realize the gas network simulation, the Decoupled Implicit Method for Efficient Network Simulation 
(DIMENS) method, which was proposed in our previous papers [37, 38], is adopted. By this method, the flow governing equations (Eqs. (7)-(9), (13) and (15)-(16)) and the thermal governing equations (Eqs. (10)-(12), (14) and (17)) are solved separately, as shown in Figure 2. The hydraulic-thermal state of the network can be depicted efficiently and accurately.

To generate and select operation schemes, this paper adopts a hybrid evolutionary algorithm based on the Elitist Non-dominated Sorting method [40] and Self-adapting Differential Evolution (saDE) algorithm [20, 22], which is named NS-saDE algorithm. This algorithm shows excellent convergence performance [41] and overcomes the difficulty in determining the value of algorithm control parameters. The NS-saDE algorithm mainly includes the following parts: Initialization, saDE mutation, saDE crossover, calculation of objective functions, sharing fitness, non-dominated sorting, crowding distance assignment and selection, as shown in Figure 3. For the constraints in Section 3.2, the restriction of decision variables is reflected in the initialization and saDE mutation. The other constraints in Section 3.2 are limited by the non-dominated sorting, which can exclude the operation schemes that dissatisfy them.

\section{Results and discussion}

\subsection{Case description}

In this paper, a large-scale natural gas transmission network with a complex topology is considered and shown in Figure 4. The pipeline network is composed of 4 sources, 21 consumers, 42 pipelines, 4 compressors and 5 valves.

\section{Pipeline parameters}

All pipes are horizontal with a roughness and wall thickness of respectively $0.23 \mathrm{~mm}$ and $8 \mathrm{~mm}$. The serial number, length and external diameter of each pipeline can be seen in Appendix Table A1. The allowable pressure of a pipe is between 3.5 and 7.0 MPa, which is also applicable to compressors and valves. The minimum outlet temperature of pipe is $278.15 \mathrm{~K}$.

The standard pressure and temperature are $101.325 \mathrm{kPa}$ and $293.15 \mathrm{~K}$, respectively. The total heat transfer coefficient is $1.5 \mathrm{~W} /\left(\mathrm{m}^{2} \mathrm{~K}\right)$, and the environmental temperature is maintained at $293.15 \mathrm{~K}$.

For a pipeline, the failure probability within a period can be obtained by the following formula:

$$
F=1-\mathrm{e}^{-\delta_{p} L t}
$$

where $\delta_{p}=1.46 \times 10^{-8} /(\mathrm{km} \mathrm{h})[29]$.

\section{Compressor parameters}

For each station, there is one compressor in operation, with the rotational speed adjustable between 4400 and $7200 \mathrm{r} / \mathrm{min}$. The maximum outlet temperature of compressor is $328.15 \mathrm{~K}$. The serial number of each compressor can

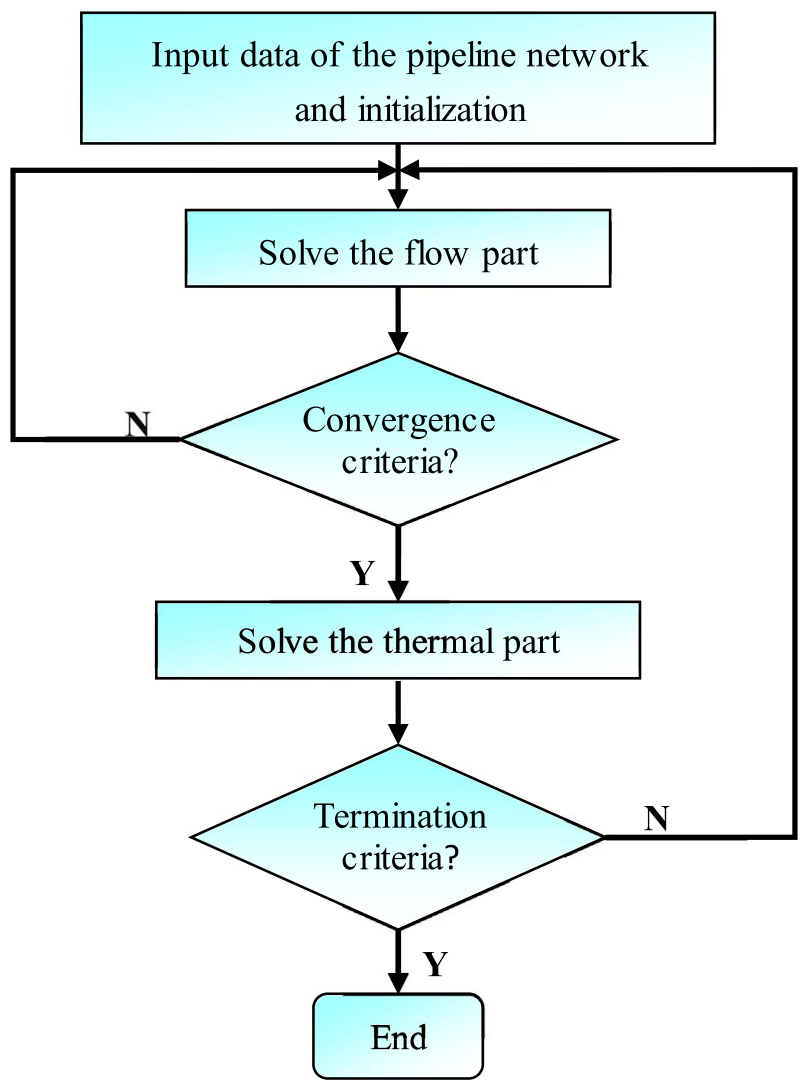

Fig. 2. The DIMENS method.

be seen in Appendix Table A2. According to the performance data given in Appendix Table A3, the fitting formula of compression ratio $e$ and inlet flow $Q_{\text {in }}$ is shown as,

$$
\varepsilon\left(Q_{\text {in }}, n\right)=a_{0} Q_{\text {in }}^{2}+b_{0} Q_{\text {in }}+c_{0},
$$

where the coefficients $a_{0}, b_{0}$ and $c_{0}$ are fitted with rotational speed by optimal square approximation:

$$
\begin{aligned}
& a_{0}=a_{1} n^{2}+a_{2} n+a_{3}, \\
& b_{0}=b_{1} n^{2}+b_{2} n+b_{3}, \\
& c_{0}=c_{1} n^{2}+c_{2} n+c_{3} .
\end{aligned}
$$

In addition, the efficiency of compressor $n\left(Q_{\text {in }}, n\right)$ can also be obtained by using the above fitting formulas. The surge flow $Q^{\min }(n)$ and block flow $Q^{\max }(n)$ are fitted with rotational speed by optimal square approximation, respectively.

\section{Valve parameters}

The network has five valves, and the serial number of each valve can be seen in Appendix Table A4. The valve opening varies from 0.5 to 1.0 . It is assumed that the flow 


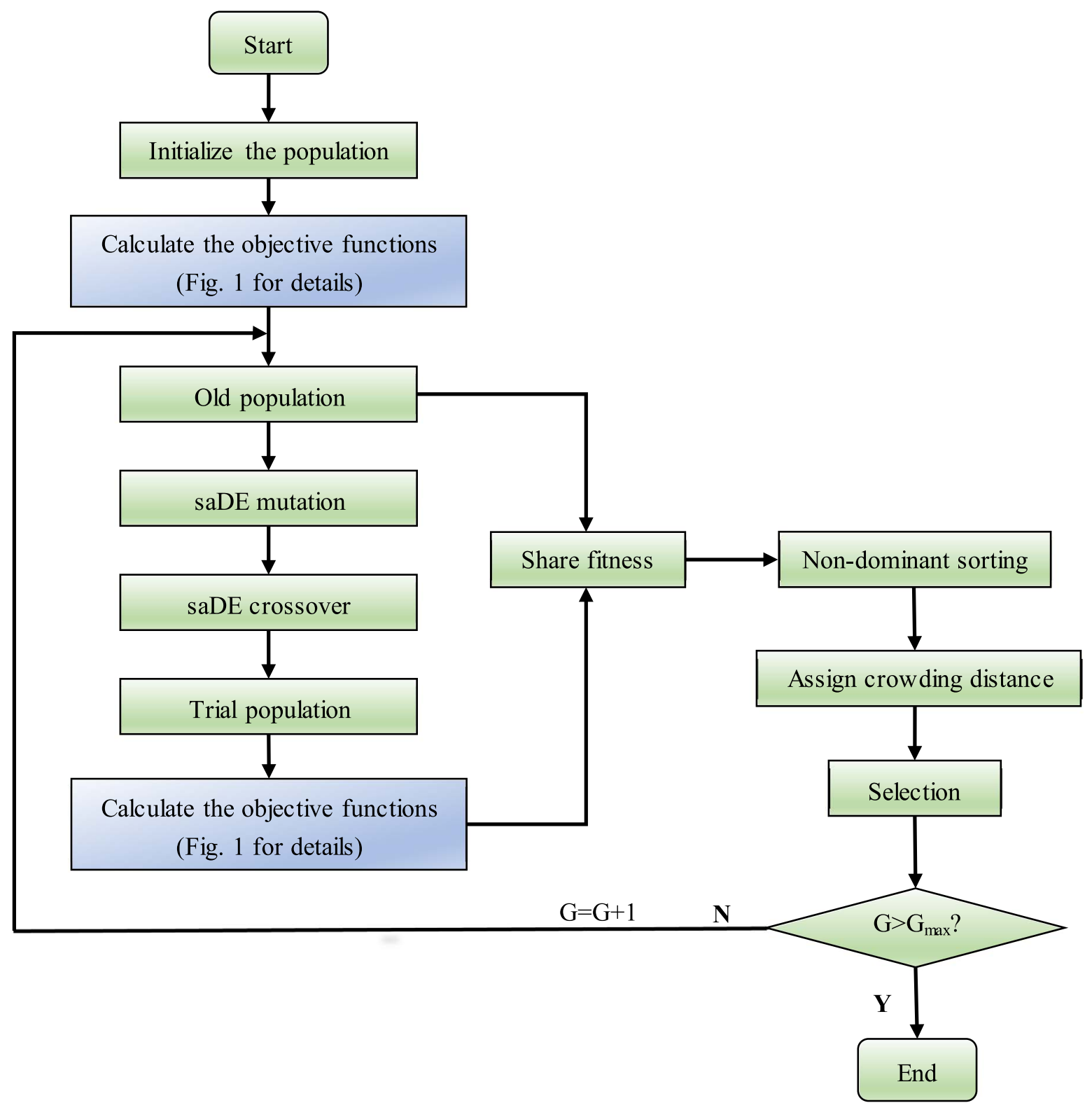

Fig. 3. The NS-saDE algorithm.

coefficient of the valve FR is linearly related with the valve opening, having,

$$
\mathrm{FR}=\frac{C v_{\max }-C v}{C v_{\max }-C v_{\min }},
$$

where $F R$ is the flow coefficient of the valve, $C v_{\max }$ is $250 \mathrm{~m}^{3} /(\mathrm{h} \mathrm{kPa})$ and $C v_{\min }$ is 0 . The valve failure is considered to happen $0.5 \times 10^{-3}$ times per year.

\section{Boundary parameters}

The network has 21 consumers and 4 sources, and the serial number of each boundary node can be seen in Appendix Table A5. Each inlet of a compressor corresponds to a source, and all sources are at 5.0 MPa and $313.15 \mathrm{~K}$. The components of natural gas in a source are listed in Appendix Table A6. The design flow rate of consumers is listed in Appendix Table A7. For all consumers, the minimum supply pressure and required pressure are $0.1 \mathrm{MPa}$ and 4.0 MPa respectively.

A real large-scale pipeline network has several transmission schemes with set loads. To reduce the computation burden, transmission coefficient with discrete values is used, and they are $\beta \in\{0.6,0.65,0.7 \ldots 1.05,1.1\}$.

\subsection{Analysis of results}

For each transmission coefficient which belongs to $\{0.6$, $0.65,0.7 \ldots 1.05,1.1\}$, the population size is set as 100 , and the number of iterations is set as 300 . The calculation 


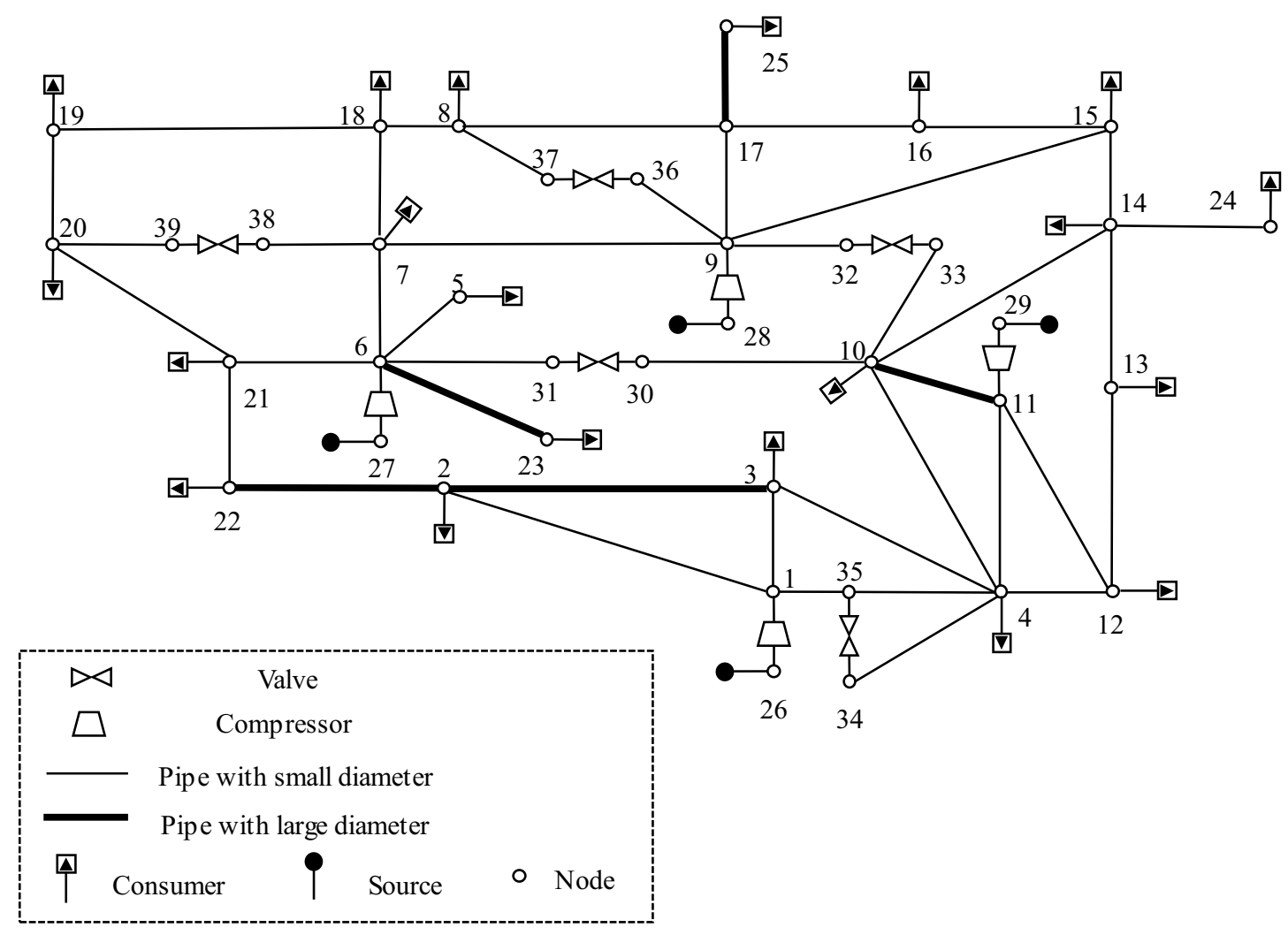

Fig. 4. The schematic diagram of the pipeline network.

was executed on a PC with Xeon W-2125 4.00 GHz processor and 16 GB RAM. Each transmission coefficient took about 1.5 million times of pipeline network simulation, which costs $11.5 \mathrm{~h}$ of computation time. To demonstrate the practical effect brought by the multi-objective optimization model considering pipeline network reliability, the Pareto fronts, the extreme value of objective properties, the consumer reliability and the impact of pipeline failure are analyzed.

\subsubsection{Analysis of Pareto fronts}

As mentioned earlier, pipeline network reliability and power cost need to be compromised, which is also the purpose of the multi-objective optimization and benefits pipeline management. The profiles of reliability and the total power of compressors for representative transmission coefficient are shown in Figure 5, which are Pareto fronts that reflect the relationship between the two objectives. Analyzing the Pareto fronts can evaluate the variation of pipeline network reliability along with an increased power and assist decision-making in selecting the final operation scheme.

Figure 5 shows increasing pipeline network reliability as the power is improved, which can be explained as follows. The compression ratio is larger for increased compressor power, and the pressure inside the pipeline network increases, meaning higher hydraulic availability of consumers and better pipeline network reliability. The observations in Figure 5 are consistent with reports in previous literature $[26,28]$. Su et al. [26] proposed that enhancing the power would reduce the interruption probability, and Shinstine et al. [28] confirmed larger system reliability when the pressure at consumer nodes is higher. The accordance of our present results with previous researches verifies the correctness of the model.

In Figure 5, it is also indicated that the detailed variation of pipeline network reliability against the compressor power is different under different transmission coefficients. When the transmission coefficient is small, the reliability displays a gentle increase with the increase of the compressor power. However, in the cases of large transmission coefficients, pipeline network reliability improves significantly with the increasing power. For example, for $\beta=1.05$, increasing the power by $5.26 \mathrm{MW}$ contributes to an increase in the reliability from 0.933 to 0.971 , which greatly guarantees the gas supply of the pipeline network. It is inferred that improving the compressor power is a feasible way to strengthen the gas supply reliability. However, it can be noted that for cases such as $\beta=0.80$ and 0.90 , the network reliability undergoes a fast rising stage followed by an almost unchanging stage in the process of increasing the compressor power. That is, sometimes continuing to increase the compressor power may produce little effect on improving the pipeline network reliability. Overall the 


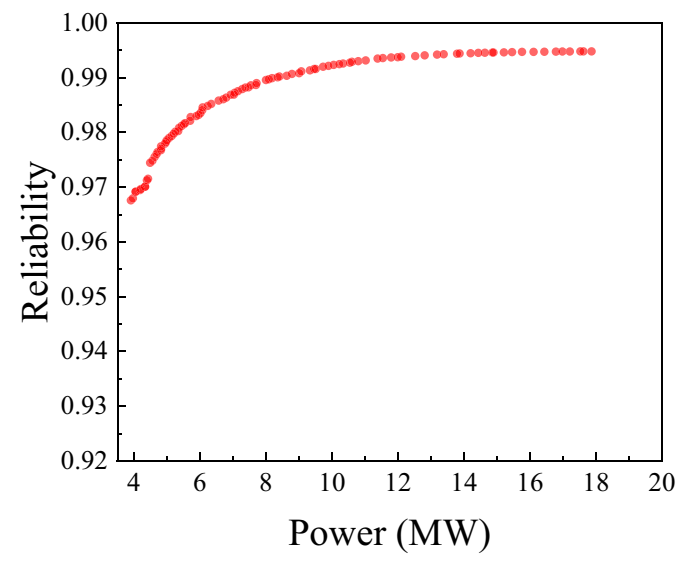

(a) $\beta=0.80$

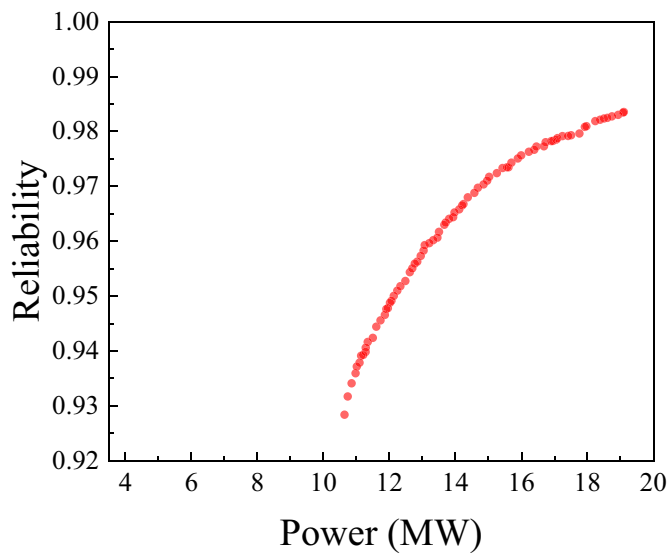

(c) $\beta=1.0$

Fig. 5. Pareto fronts under different transmission coefficients.

transmission system operator can select the appropriate operation schemes in different situations through the Pareto fronts. For example, if the gas supply is significant to the regional energy supply, the operator can choose an operation scheme with high reliability, otherwise the low power consumption scheme can be adopted to save cost. By the multi-objective optimization model, a wide range of options can be provided for the operator, which are satisfactory in both reliability and economy.

\subsubsection{Analysis of extreme values}

The influence of throughput on the pipeline network reliability and compressor power can be analyzed from the perspective of extreme values of the objective properties. The maximum and minimum pipeline network reliability under different transmission coefficients are shown in Figure 6 . Similarly, the distributions of the maximum and minimum power are presented in Figure 7.

On the whole, Figure 6 illustrates decreasing pipeline network reliability as the throughput becomes larger, which

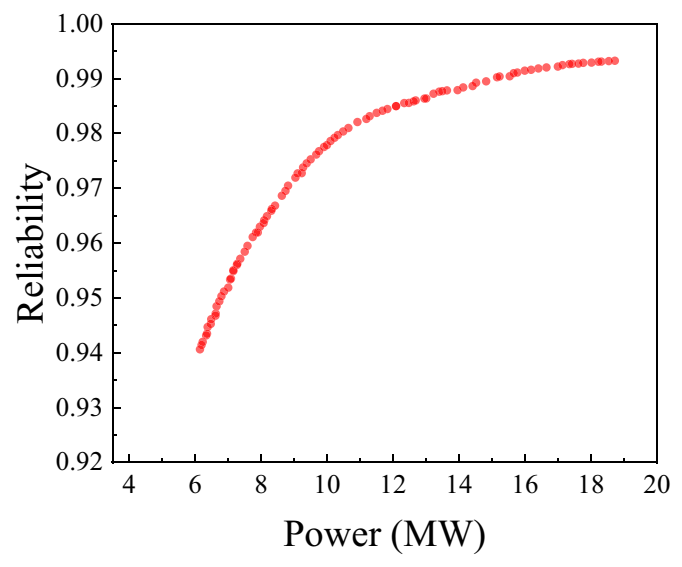

(b) $\beta=0.90$

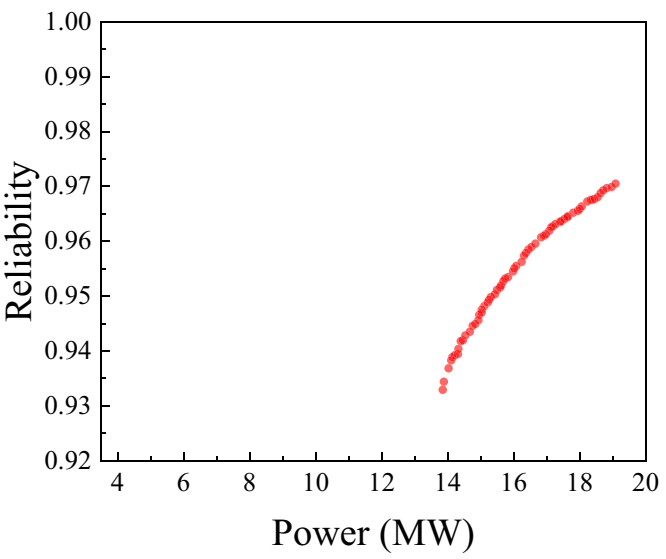

(d) $\beta=1.05$

is in accordance with the result of Shinstine et al. [28], that the reliability is a negative function of flow rate. Figure 7 shows that with the increase in the throughput, the power consumption increases, which is consistent with the multiobjective optimization results of Botros et al. [9] and Yang et al. [13].

For transmission coefficient varying from 1.0 to 1.10 , the maximum pipeline network reliability declines sharply, as is demonstrated in Figure 6. It is illustrated that in such situations, further increasing the throughput is not beneficial to guaranteeing gas supply. While correspondingly, Figure 7 shows a rapid rise of the minimum power for $\beta$ from 1.0 to 1.10 , meaning that the transmission operation should be conducted under-high power schemes which naturally leads to higher reliability, explaining the rise of minimum pipeline network reliability observed in Figure 6 . For the extreme throughput of this network, that is $\beta=1.10$, the maximum and minimum power are almost the same. For transmission coefficients between 0.6 and 0.7 , the reliability of the pipe network maintains at a high value of 0.995, and the variation of throughput brings insignificant 


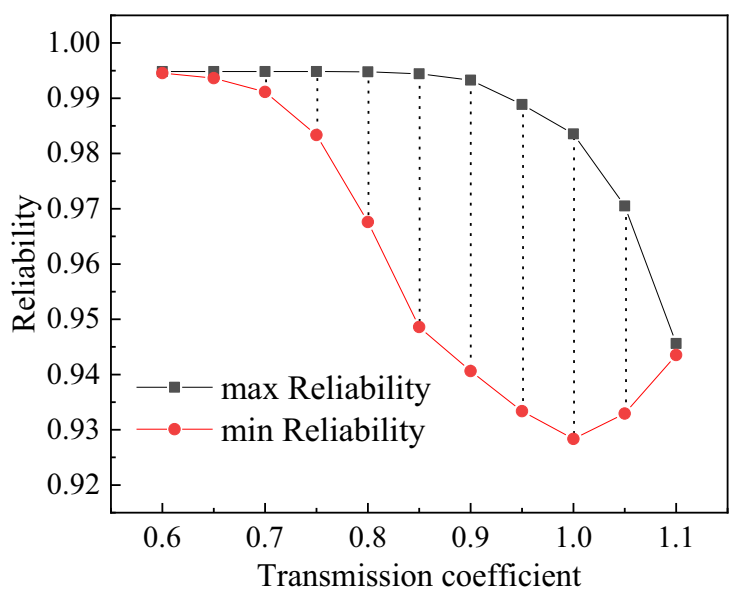

Fig. 6. The extreme values of pipeline network reliability.

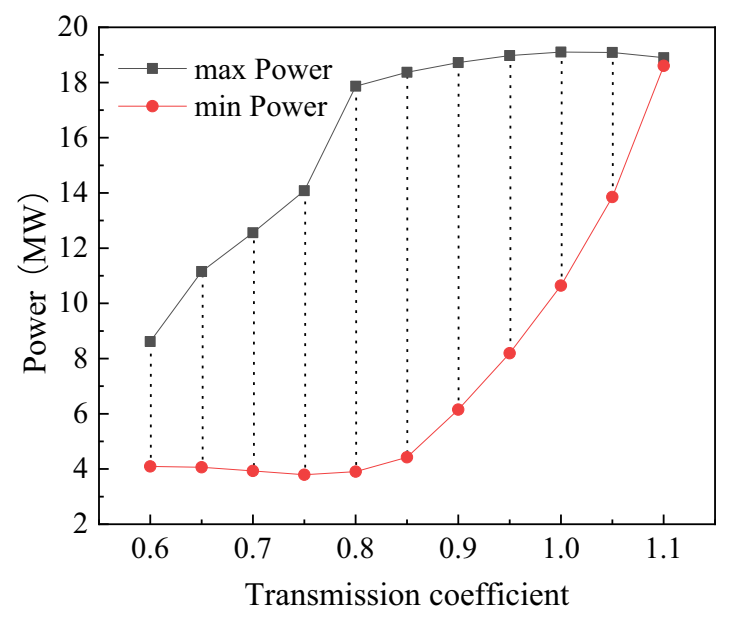

Fig. 7. The extreme values of compressor power.

change of the reliability. From the above analysis it is known that the multi-objective optimization model can evaluate the relationship between pipeline network reliability and throughput.

\subsubsection{Analysis of consumer reliability}

Consumer reliability can quantitatively reflect the probability that a consumer obtains the demanded flow rate. Consumer nodes that are susceptible to fault state can be identified by analyzing the consumer reliability of each consumer node based on the optimization results. For each transmission coefficient, the consumer reliability of each consumer node is averaged, and the results are shown in Figure 8.

Observing Figure 8, one can notice that the consumer reliability depends greatly on the transmission coefficient. The larger transmission coefficient is, on the whole the lower the consumer reliability becomes. Consumer nodes with low consumer reliability have lower probability of receiving the required flow rate. Significantly, for large transmission coefficients, consumers No. 13, No. 24, and No. 25 show low reliability than others. The system operator should give prior monitoring to the three consumers, and gas supply can be guaranteed by other ways such as compressed natural gas vehicles. Comparatively, consumers No. 4, No. 7 and No. 10 always have high consumer reliability, indicating strong resistance to the failure situations of the pipeline network. In short summary, the multi-objective optimization model can analyze the ability of a pipeline network to deliver required flow rate to individual consumers.

\subsubsection{Analysis of the impact of pipeline failure}

The impact of failure of a pipeline on the overall transmission of the pipeline network can be investigated from the perspective of the minimum cut set. The minimum cut set of a consumer node refers to a set of pipelines that once encounter failure status leads to inability of satisfying the consumer's demand. In the optimization process, the iteration results approach the Pareto front gradually. The results of the last several iterations can be seen as operation schemes balancing the pipeline network reliability and power, which are the possible operating status of the pipeline network. The minimum cut sets appearing in the last three iterations are selected, and the percentage of consumers in failure caused by the break of each pipeline is calculated respectively, as presented in Figure 9.

It can be observed from Figure 9 that when the transmission coefficient increases, the consumers in failure account for a larger percentage, which causes lower pipeline network reliability. Also the percentage of consumers in failure caused by the pipe can reflect to what extent the failure of this pipeline disturbs gas supply of the pipeline network. For instance, when pipe No. 15 is in failure for $\beta=1.1,88 \%$ of the consumers cannot reach the required flow rate. So the system operator should place more sensors to strengthen the monitoring and enhance the maintenance for this pipeline to reduce its failure possibility. However, the failure of pipe No. 37 only gives rise to $10 \%$ consumer failure, indicating that the failure of this pipeline produces little impact on the gas supply system. Thus the multi-objective optimization model can judge the influence of a single pipeline on the whole pipeline network, and provide valuable guidance in taking proper measures.

\section{Conclusion}

Reducing the operating cost has been the pursuit of previous optimization researches of gas pipeline networks, which however would lead to a decline in the pipeline network reliability. To achieve trade-off between reliability and operating cost, a multi-objective optimization model is developed in this study to determine optimal operation scheme for natural gas pipeline network. This model 


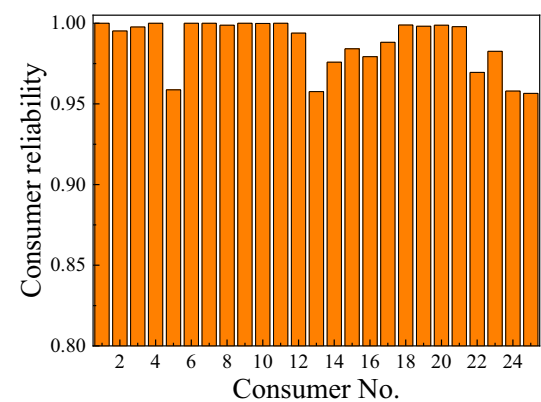

(a) $\beta=0.80$

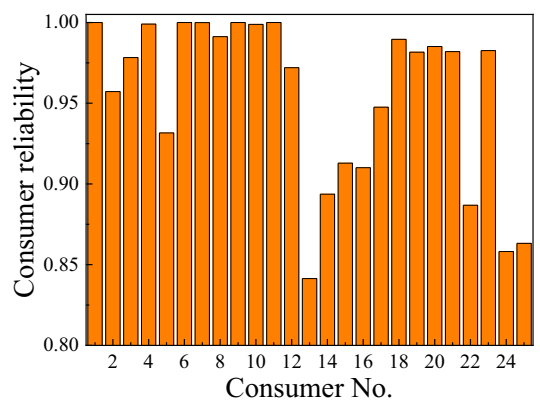

(c) $\beta=1.2$

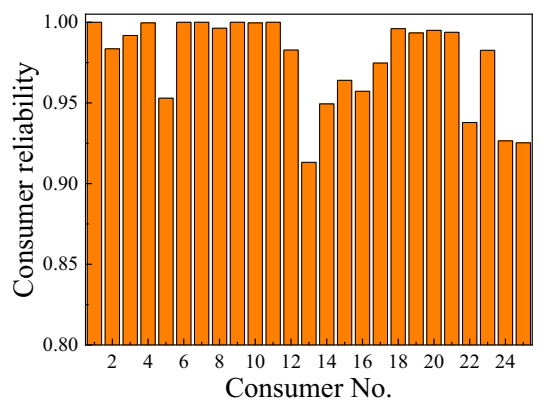

(b) $\beta=0.9$

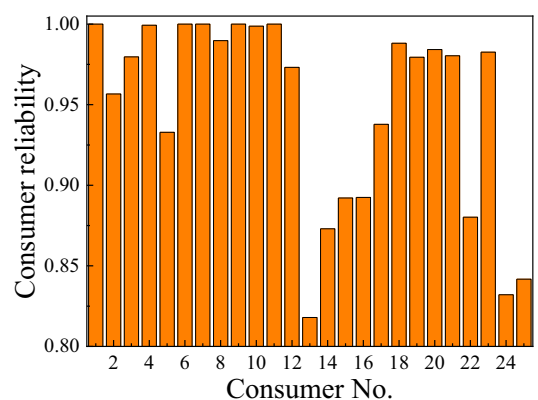

(d) $\beta=1.05$

Fig. 8. Consumer reliability under different transmission coefficients.

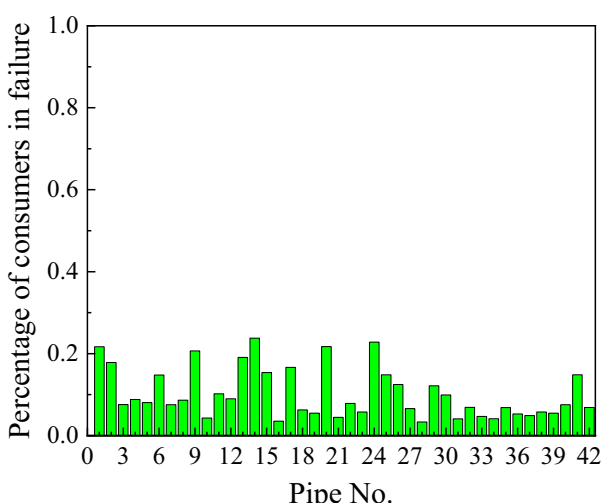

(a) $\beta=0.80$

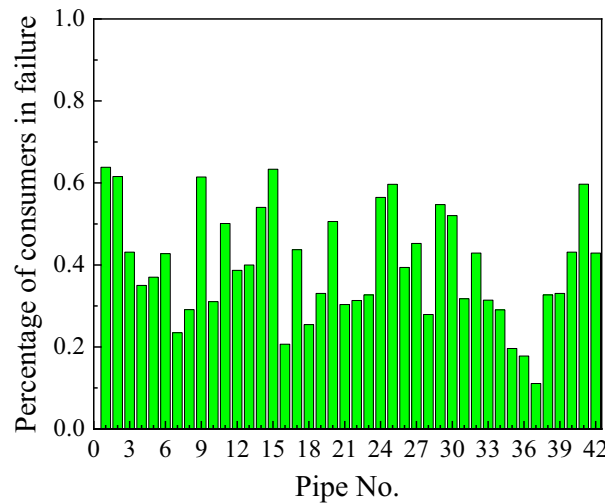

(c) $\beta=1.0$

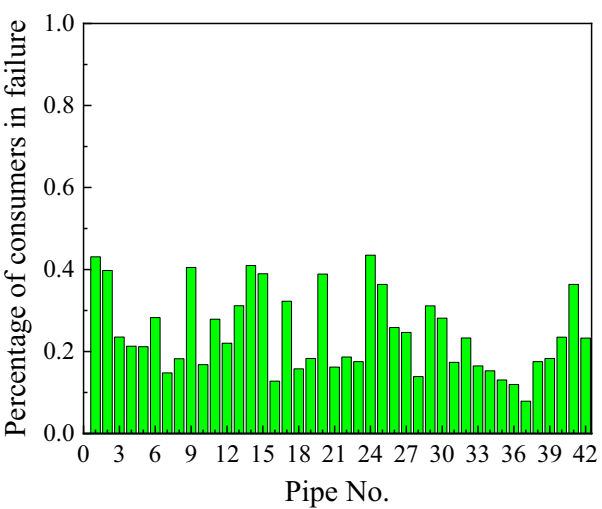

(b) $\beta=0.90$

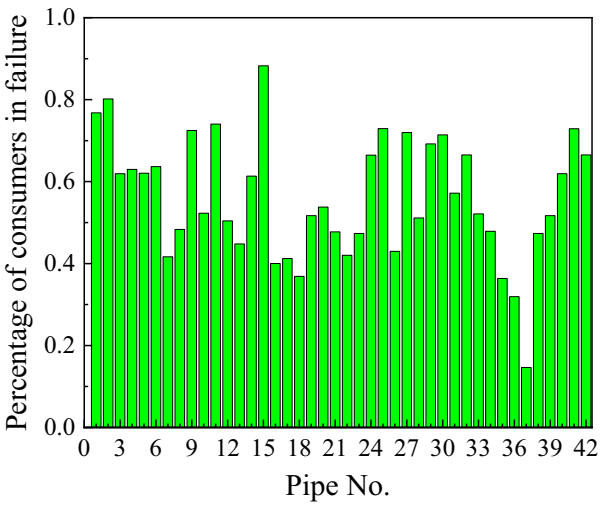

(d) $\beta=1.10$

Fig. 9. Percentage of consumers in failure caused by the break of a pipe under different transmission coefficients. 
considers a pipeline network with multiple components and a complicated topology. The evaluation of pipeline reliability takes the failure of each component and the hydraulicthermal state of the fault pipeline network into consideration. The optimization variables are the rotating speed of the compressors and the opening of the valves. The established multi-objective optimization model can be solved effectively by DIMENS method and NS-saDE algorithm. Application of this proposed model is analyzed in a complicated natural gas pipeline network, by which the Pareto solution set and extreme values of the two optimal variables are discussed under different throughputs. There are the following conclusions:

1. The Pareto solution set obtained by the proposed model is able to reflect the quantitative correlations between pipeline network reliability and the total power. For different throughputs, larger total power results in higher pipeline network reliability. In cases of throughputs lower than rated load, pipeline network reliability is reduced to a small extent due to the reduced compressor power. While when the pipeline transports at a load larger than rated, a small decrease of compressor power significantly reduces the pipeline network reliability. The Pareto solution set assists to determine the operation scheme providing trade-off between pipeline network reliability and the total power.

2. The extreme values of the optimal variables stand for the maximum and minimum pipeline network reliability and operating cost under different throughputs. The limited throughput can be determined accordingly. When the network transports at a small load, the reliability is relatively high and is insensitive to the variation of compressor power. As the throughput increases, the maximum value of pipeline network reliability drops rapidly and the minimum value of compressor power rises rapidly. At the extreme throughput, high-power is required to ensure the normal operation of the pipeline network, which corresponds to the lowest reliability and the largest operating cost.

3. The probability of each consumer obtaining the demanded gas distribution can be quantitatively obtained by the model, which acts as a basis for determining the consumers for prior maintenance. At low throughput, the reliability of each customer is relatively high. With the increase in throughput, the reliability of some customers drops significantly, and these consumers are selected to give prior attention. Strengthening the minoring and maintenance of these consumers can effectively improve the pipeline network reliability.

4. When a pipeline is in failure, the percentage of consumers failing to get demanded gas supply can be calculated by the proposed model to evaluate the failure of this pipeline on the whole transmission. The pipelines, which when fail result in larger percentage of consumers in failure, should be given more attention to enhance the pipeline network reliability.
Acknowledgments. The study is supported by the National Natural Science Foundation of China (No. 51806018), Project of Construction of Innovative Teams and Teacher Career Development for Universities and Colleges under Beijing Municipality (No. IDHT20170507) and the Program of Great Wall Scholar (No. CIT\&TCD20180313).

\section{References}

1 BP (2019) BP statistical review of world energy-Natural gas, Technical Report British Petroleum (BP).

2 Üster H., Dilaveroğlu Ş. (2014) Optimization for design and operation of natural gas transmission networks, Appl. Energy 133, 56-69.

3 Di Lullo G., Oni A.O., Gemechu E., Kumar A. (2020) Developing a greenhouse gas life cycle assessment framework for natural gas transmission pipelines, J. Nat. Gas Sci. Eng. $\mathbf{7 5}, 103136$

4 Demissie A., Zhu W., Belachew C.T. (2017) A multiobjective optimization model for gas pipeline operations, Comput. Chem. Eng. 100, 94-103.

5 Saidur R., Rahim N.A., Hasanuzzaman M. (2010) A review on compressed-air energy use and energy savings, Renew. Sustain. Energy Rev. 14, 1135-1153.

6 Ahmadian Behrooz H. (2016) Managing demand uncertainty in natural gas transmission networks, J. Nat. Gas Sci. Eng. 34, 100-111.

7 Mikolajková M., Saxén H., Pettersson F. (2018) Linearization of an MINLP model and its application to gas distribution optimization, Energy 146, 156-168.

8 Liang Y., Hui C. (2018) Convexification for natural gas transmission networks optimization, Energy 158, 1001-1016.

9 Botros K., Sennhauser D., Jungowski K., Poissant G., Golshan H., Stoffregen J. (2004) Multi-objective optimization of large pipeline networks using genetic algorithm, in: International Pipeline Conference, Calgary, Canada, pp. 2005-2015.

10 Kashani A.H.A., Molaei R. (2014) Techno-economical and environmental optimization of natural gas network operation, Chem. Eng. Res. Des. 92, 2106-2122.

11 Panda D., Ramteke M. (2019) Preventive crude oil scheduling under demand uncertainty using structure adapted genetic algorithm, Appl. Energy 235, 68-82.

12 Fettaka S., Thibault J. (2013) Pipeline optimization using a novel hybrid algorithm combining front projection and the non-dominated sorting genetic algorithm-II (FP-NSGA-II), in: IEEE Congress on Evolutionary Computation, Cancun, Mexico, pp. 697-704.

13 Yang Y., Liu J., Tan S., Wang H. (2018) Application of constrained multi-objective evolutionary algorithm in multisource compressed-air pipeline optimization problems, IFAC-PapersOnLine 51, 168-173.

$14 \mathrm{Hu}$ Y., Bie Z., Ding T., Lin Y. (2016) An NSGA-II based multi-objective optimization for combined gas and electricity network expansion planning, Appl. Energy 167, 280-293.

15 Qu K., Yu T., Zhang X., Li H. (2019) Homogenized adjacent points method: A novel Pareto optimizer for linearized multiobjective optimal energy flow of integrated electricity and gas system, Appl. Energy 233-234, 338-351.

16 Zheng J., Wu Q., Jing Z. (2017) Coordinated scheduling strategy to optimize conflicting benefits for daily operation of integrated electricity and gas networks, Appl. Energy 192, $370-381$. 
17 Zan T.T.T., Gupta P., Wang M., Dauwels J., Ukil A. (2018) Multi-objective optimal sensor placement for low-pressure gas distribution networks, IEEE Sens J. 18, 6660-6668.

18 Sheikh Alivand M., Farhadi F. (2018) Multi-objective optimization of a multi-layer PTSA for LNG production, $J$. Nat. Gas Sci. Eng. 49, 435-446.

19 Wang X., Zhou F., Xia T., Xu M. (2016) A multi-objective optimization model to enhance the comprehensive performance of underground gas drainage system, J. Nat. Gas Sci. Eng. 36, 852-864.

20 Vesterstrom J., Thomsen R. (2004) A comparative study of differential evolution, particle swarm optimization, and evolutionary algorithms on numerical benchmark problems, in: IEEE Congress on Evolutionary Computation, Portland, USA, pp. 1980-1987.

21 Das S., Suganthan P.N. (2011) Differential evolution: A survey of the state-of-the-art, IEEE Trans Evol. Comput. 15, 4-31.

22 Brest J., Greiner S., Boskovic B., Mernik M., Zumer V. (2006) Self-adapting control parameters in differential evolution: A comparative study on numerical benchmark problems, IEEE Trans Evol. Comput. 10, 646-657.

23 Yazdi J., Choi Y.H., Kim J.H. (2017) Non-dominated sorting harmony search differential evolution (NS-HS-DE): A hybrid algorithm for multi-objective design of water distribution networks, Water 9, 587. https://doi.org/10.3390/w9080587.

24 Ewees A.A., Abd Elaziz M., Oliva D. (2021) A new multiobjective optimization algorithm combined with oppositionbased learning, Expert Syst. Appl. 165, 113844.

$25 \mathrm{Yu}$ W., Wen K., Li Y., Huang W., Gong J. (2018) A methodology to assess the gas supply capacity and gas supply reliability of a natural gas pipeline network system, in: International Pipeline Conference, Calgary, Canada.

$26 \mathrm{Su} \mathrm{H.,} \mathrm{Zio} \mathrm{E.,} \mathrm{Zhang} \mathrm{J.,} \mathrm{Li} \mathrm{X.,} \mathrm{Chi} \mathrm{L.,} \mathrm{Fan} \mathrm{L.,} \mathrm{Zhang} \mathrm{Z.}$ (2019) A method for the multi-objective optimization of the operation of natural gas pipeline networks considering supply reliability and operation efficiency, Comput. Chem. Eng. 131, 106584.

27 Yu W., Gong J., Song S., Huang W., Li Y., Zhang J., Hong B., Zhang Y., Wen K., Duan X. (2019) Gas supply reliability analysis of a natural gas pipeline system considering the effects of underground gas storages, Appl. Energy 252, 113418.

28 Shinstine D.S., Ahmed I., Lansey K.E. (2002) Reliability/ availability analysis of municipal water distribution networks: case studies, J. Water Resour. Plan. Manag. 128, 140-151.
29 Yu W., Song S., Li Y., Min Y., Huang W., Wen K., Gong J. (2018) Gas supply reliability assessment of natural gas transmission pipeline systems, Energy 162, 853-870.

30 Li J., Liu W. (2006) Large-scale urban network seismic reliability analysis and optimization, Earthq. Eng. Eng. Vib. 26, 172-175 (in Chinese).

31 Shabazbegian V., Ameli H., Ameli M.T., Strbac G. (2020) Stochastic optimization model for coordinated operation of natural gas and electricity networks, Comput. Chem. Eng. 142, 107060.

32 Zheng F., Simpson A.R., Zecchin A.C. (2013) A decomposition and multistage optimization approach applied to the optimization of water distribution systems with multiple supply sources, Water Resour Res. 49, 380-399.

33 Su Y., Mays L.W., Duan N., Lansey K.E. (1987) Reliabilitybased optimization model for water distribution systems, J. Hydraul. Eng. 113, 1539-1556.

34 Li J., Qin C., Yan M., Ma J., Yu J. (2016) Hydraulic reliability analysis of an urban loop high-pressure gas network, J. Nat. Gas Sci. Eng. 28, 372-378.

35 Saeid M., Poe W.A. (2012) Sales gas transmission, in: Handbook of natural gas transmission and processing (second edition), Gulf Professional Publishing, Boston, pp. 425-450.

36 Liu E., Lv L., Yi Y., Xie P. (2019) Research on the steady operation optimization model of natural gas pipeline considering the combined operation of air coolers and compressors, IEEE Access 7, 83251-83265.

37 Wang P., Ao S., Yu B., Han D., Xiang Y. (2019) An efficiently decoupled implicit method for complex natural gas pipeline network simulation, Energies 12.

38 Wang P., Yu B., Han D., Sun D., Xiang Y. (2018) Fast method for the hydraulic simulation of natural gas pipeline networks based on the divide-and-conquer approach, J. Nat. Gas Sci. Eng. 50, 55-63.

39 Benedict M., Webb G.B., Rubin L.C. (1940) An empirical equation for thermodynamic properties of light hydrocarbons and their mixtures I. Methane, Ethane, Propane and n-Butane, J. Chem. Phys. 8, 334-345.

40 Deb K., Pratap A., Agarwal S., Meyarivan T. (2002) A fast and elitist multiobjective genetic algorithm: NSGA-II, IEEE Trans, Evol. Comput. 6, 182-197.

41 Yuan Q., Li J., Liu H., Yu B., Sun D., Deng Y. (2019) Parametric regression of a multiparameter thixotropic model for waxy crude oil based on multiobjective strategy, J. Pet. Sci. Eng. 173, 287-297. 


\section{Appendix}

Table A1. Pipeline data.

\begin{tabular}{lccccccccccccccccccccc}
\hline Serial number & 1 & 2 & 3 & 4 & 5 & 6 & 7 & 8 & 9 & 10 & 11 & 12 & 13 & 14 & 15 & 16 & 17 & 18 & 19 & 20 & 21 \\
\hline Starting node & 1 & 1 & 34 & 4 & 3 & 2 & 5 & 22 & 6 & 4 & 4 & 4 & 12 & 12 & 11 & 13 & 10 & 14 & 9 & 15 & 15 \\
Ending node & 2 & 3 & 4 & 3 & 2 & 22 & 6 & 21 & 21 & 10 & 11 & 12 & 13 & 11 & 10 & 14 & 14 & 15 & 32 & 9 & 16 \\
Length (km) & 48 & 50 & 70 & 60 & 80 & 90 & 120 & 104 & 60 & 80 & 70 & 110 & 70 & 60 & 80 & 80 & 150 & 160 & 100 & 160 & 150 \\
External diameter (mm) & 406 & 406 & 406 & 406 & 508 & 508 & 406 & 406 & 406 & 406 & 406 & 406 & 406 & 406 & 508 & 406 & 406 & 406 & 406 & 406 & 406 \\
\hline Serial number & 22 & 23 & 24 & 25 & 26 & 27 & 28 & 29 & 30 & 31 & 32 & 33 & 34 & 35 & 36 & 37 & 38 & 39 & 40 & 41 & 42 \\
\hline Starting node & 16 & 10 & 9 & 9 & 8 & 9 & 8 & 7 & 6 & 18 & 7 & 20 & 20 & 6 & 14 & 17 & 31 & 33 & 1 & 37 & 39 \\
Ending node & 17 & 30 & 17 & 36 & 17 & 7 & 18 & 18 & 7 & 19 & 38 & 19 & 21 & 23 & 24 & 25 & 6 & 10 & 35 & 8 & 20 \\
Length (km) & 160 & 100 & 130 & 80 & 110 & 90 & 60 & 84 & 40 & 60 & 80 & 64 & 90 & 40 & 40 & 40 & 60 & 100 & 70 & 80 & 80 \\
External diameter (mm) & 406 & 406 & 406 & 406 & 406 & 406 & 406 & 406 & 406 & 406 & 406 & 406 & 406 & 508 & 406 & 508 & 406 & 406 & 406 & 406 & 406 \\
\hline
\end{tabular}

Table A2. Compressor data.

\begin{tabular}{lcccc}
\hline Serial number & 1 & 2 & 3 & 4 \\
\hline Starting node & 26 & 27 & 28 & 29 \\
Ending node & 1 & 6 & 9 & 11 \\
\hline
\end{tabular}


Table A3. Performance data of compressor.

\begin{tabular}{|c|c|c|c|c|c|c|c|c|c|}
\hline$H(\mathrm{~m})$ & $\eta$ & $Q_{\text {in }}\left(\mathrm{am}^{3} / \mathrm{h}\right)$ & $\varepsilon$ & $n(\mathrm{r} / \mathrm{min})$ & $H(\mathrm{~m})$ & $\eta$ & $Q_{\text {in }}\left(\mathrm{am}^{3} / \mathrm{h}\right)$ & $\varepsilon$ & $n(\mathrm{r} / \mathrm{min})$ \\
\hline 2080 & 0.87 & 3741 & 1.15 & 4412 & 4039 & 0.88 & 5369 & 1.3 & 6109 \\
\hline 2024 & 0.87 & 4026 & 1.14 & 4412 & 3876 & 0.88 & 6000 & 1.29 & 6109 \\
\hline 1927 & 0.88 & 4534 & 1.14 & 4412 & 3744 & 0.88 & 6495 & 1.28 & 6109 \\
\hline 1802 & 0.88 & 5029 & 1.13 & 4412 & 3600 & 0.88 & 6978 & 1.26 & 6109 \\
\hline 1691 & 0.88 & 5536 & 1.12 & 4412 & 3243 & 0.87 & 7981 & 1.24 & 6109 \\
\hline 1559 & 0.87 & 6006 & 1.11 & 4412 & 3054 & 0.86 & 8513 & 1.22 & 6109 \\
\hline 1484 & 0.86 & 6279 & 1.1 & 4412 & 2874 & 0.86 & 8959 & 1.21 & 6109 \\
\hline 1372 & 0.84 & 6650 & 1.1 & 4412 & 2677 & 0.84 & 9442 & 1.19 & 6109 \\
\hline 1269 & 0.82 & 7008 & 1.09 & 4412 & 2451 & 0.82 & 9975 & 1.17 & 6109 \\
\hline 1130 & 0.8 & 7441 & 1.08 & 4412 & 2146 & 0.79 & 10619 & 1.15 & 6109 \\
\hline 2425 & 0.87 & 4044 & 1.17 & 4751 & 4963 & 0.88 & 6235 & 1.37 & 6788 \\
\hline 2304 & 0.88 & 4589 & 1.17 & 4751 & 4757 & 0.88 & 6988 & 1.36 & 6788 \\
\hline 2202 & 0.88 & 5035 & 1.16 & 4751 & 4420 & 0.88 & 8000 & 1.33 & 6788 \\
\hline 2081 & 0.88 & 5542 & 1.15 & 4751 & 4230 & 0.88 & 8531 & 1.31 & 6788 \\
\hline 1955 & 0.88 & 6025 & 1.14 & 4751 & 4055 & 0.87 & 8988 & 1.3 & 6788 \\
\hline 1844 & 0.87 & 6420 & 1.13 & 4751 & 3801 & 0.87 & 9568 & 1.28 & 6788 \\
\hline 1656 & 0.85 & 7014 & 1.12 & 4751 & 3618 & 0.86 & 9988 & 1.26 & 6788 \\
\hline 1569 & 0.84 & 7298 & 1.11 & 4751 & 3152 & 0.83 & 10988 & 1.22 & 6788 \\
\hline 1429 & 0.82 & 7682 & 1.1 & 4751 & 2858 & 0.8 & 11580 & 1.2 & 6788 \\
\hline 1313 & 0.79 & 8065 & 1.09 & 4751 & 2646 & 0.78 & 12000 & 1.18 & 6788 \\
\hline 3172 & 0.88 & 4648 & 1.23 & 5430 & 5449 & 0.88 & 6719 & 1.41 & 7127 \\
\hline 3115 & 0.88 & 4994 & 1.23 & 5430 & 5365 & 0.88 & 7037 & 1.41 & 7127 \\
\hline 2980 & 0.88 & 5501 & 1.22 & 5430 & 5038 & 0.88 & 8016 & 1.38 & 7127 \\
\hline 2858 & 0.88 & 5984 & 1.21 & 5430 & 4671 & 0.88 & 9006 & 1.35 & 7127 \\
\hline 2566 & 0.87 & 6986 & 1.18 & 5430 & 4516 & 0.87 & 9459 & 1.33 & 7127 \\
\hline 2413 & 0.86 & 7407 & 1.17 & 5430 & 4329 & 0.87 & 9997 & 1.31 & 7127 \\
\hline 2219 & 0.85 & 7976 & 1.16 & 5430 & 3828 & 0.85 & 10987 & 1.28 & 7127 \\
\hline 2024 & 0.83 & 8497 & 1.14 & 5430 & 3480 & 0.83 & 11500 & 1.25 & 7127 \\
\hline 1838 & 0.81 & 8979 & 1.13 & 5430 & 3310 & 0.82 & 11976 & 1.23 & 7127 \\
\hline 1716 & 0.79 & 9314 & 1.12 & 5430 & 2977 & 0.78 & 12685 & 1.2 & 7127 \\
\hline
\end{tabular}

Table A4. Valve data.

\begin{tabular}{lccccc}
\hline Serial number & 1 & 2 & 3 & 4 & 5 \\
\hline Starting node & 31 & 32 & 35 & 36 & 38 \\
Ending node & 30 & 33 & 34 & 37 & 39 \\
\hline
\end{tabular}

Table A5. Boundary data.

\begin{tabular}{lccccccccccccc}
\hline Serial number & 1 & 2 & 3 & 4 & 5 & 6 & 7 & 8 & 9 & 10 & 11 & 12 & 13 \\
\hline Adjacent node & $26^{*}$ & 2 & 3 & 4 & 5 & $27^{*}$ & 7 & 8 & $28^{*}$ & 10 & $29^{*}$ & 12 & 13 \\
Serial number & 14 & 15 & 16 & 17 & 18 & 19 & 20 & 21 & 22 & 23 & 24 & 25 & 25 \\
Adjacent node & 14 & 15 & 16 & 17 & 18 & 19 & 20 & 21 & 22 & 23 & 24 & 25
\end{tabular}

\footnotetext{
* The node connected with source, the others are connected with consumer.
} 
Table A6. Components of natural gas.

\begin{tabular}{lc}
\hline Component & Volume fraction (\%) \\
\hline $\mathrm{CH}_{4}$ & 96.07 \\
$\mathrm{C}_{2} \mathrm{H}_{6}$ & 1.17 \\
$\mathrm{C}_{3} \mathrm{H}_{8}$ & 0.02 \\
$\mathrm{~N}_{2}$ & 0.71 \\
$\mathrm{CO}_{2}$ & 2.03 \\
\hline
\end{tabular}

Table A7. The design flow rate of consumers.

\begin{tabular}{lccccccccccc}
\hline Serial number & 2 & 3 & 4 & 5 & 7 & 8 & 10 & 12 & 13 & 14 & 15 \\
\hline Volume flow rate $\left(10^{5} \mathrm{Nm}^{3} / \mathrm{h}\right)$ & 1.5 & 2.0 & 1.5 & 1.1 & 0.3 & 0.36 & 2 & 1.28 & 1.2 & 0.4 & 0.8 \\
Serial number & 16 & 17 & 18 & 19 & 20 & 21 & 22 & 23 & 24 & 25 & \\
Volume flow rate $\left(10^{5} \mathrm{Nm}^{3} / \mathrm{h}\right)$ & 0.4 & 0.32 & 0.8 & 0.5 & 0.6 & 0.8 & 1.5 & 1.8 & 0.4 & 1.2 & \\
\hline
\end{tabular}

